\title{
Serum Fatty Acid Binding Protein 4 Concentrations Are Positively and Independently Associated with Blood Pressure and Abdominal Fat among Parameters in Health Check-Ups in Ordinary Middle-Aged Japanese Males
}

\author{
Masami YAmadA $^{1}$, Kazuki MochizuKI ${ }^{1,2}$, Kazue Honma $^{1}$, Rie MiYauchi ${ }^{1}$, \\ Nobuhiko KASEZAWA ${ }^{3}$, Kazushige TOHYAMA ${ }^{3}$ and Toshinao GODA ${ }^{1, *}$ \\ ${ }^{1}$ Laboratory of Nutritional Physiology, University of Shizuoka, Graduate School of Nutritional and \\ Environmental Sciences, Shizuoka 422-8526, Japan \\ ${ }^{2}$ Laboratory of Food and Nutritional Sciences, Department of Local Produce and Food Sciences, Faculty of \\ Life and Environmental Sciences, University of Yamanashi, Yamanashi 400-8510, Japan \\ ${ }^{3}$ SBS Shizuoka Health Promotion Center, Shizuoka 422-8033, Japan
}

(Received June 20, 2014)

\begin{abstract}
Summary We wished to examine potential associations among blood concentrations of fatty acid-binding protein (FABP) 4 and parameters in health check-ups such as abdominal fat area (AFA) and blood pressure (BP) in middle-aged Japanese males. We conducted a cross-sectional study of males who participated in health check-ups in Japan. We excluded participants diagnosed with metabolic diseases by the time of their check-up. A total of 305 subjects (30-64 [mean \pm standard deviation, 47.3 \pm 8.5$]$ y) were recruited. Areas of totalabdominal, visceral and subcutaneous fat were measured using computed tomography. We compared the association of serum concentrations of FABP4 with various clinical parameters by Pearson product-moment correlation coefficient (PPMCC) analyses, and by stepwise multivariate linear regression analyses (MLRA). PPMCC analyses showed that blood concentrations of FABP4 were positively associated with: body mass index; areas of fat (total abdominal, visceral, subcutaneous); systolic BP; diastolic BP; total cholesterol; low-density lipoprotein-cholesterol; triacylglycerol; activities of $\gamma$-glutamyl transpeptidase, aspartate aminotransferase and alanine aminotransferase; white blood cell count; and levels of creatinine. Step-wise MLRA showed that AFAs (visceral and subcutaneous) and diastolic BP were positively and independently associated with serum concentrations of FABP4 among the parameters tested. These data suggest that serum concentrations of FABP4 are independently and positively associated with BP and AFA among parameters measured in health check-ups in middle-aged Japanese males.
\end{abstract}

Key Words FABP4, abdominal fat, Japanese males, blood pressure, CT

Recent studies have shown that fatty acid-binding protein (FABP) 4 is present in adipocytes and macrophages, and is also a novel adipocytokine secreted from adipocytes into blood (1). Animal studies have suggested that FABP4 is related to the development of insulin resistance and atherosclerosis (2-4). It has been reported that injection of a chemical inhibitor of FABP4 into animals reduced the development of atherosclerosis (5). Cross-sectional studies demonstrated blood concentrations of FABP4 to be positively associated with obesity, insulin resistance, hypertension and carotid atherosclerosis in: 234 South Korean adults; 806 American patients with type-2 diabetes mellitus (T2DM); and 459 Chinese patients who had undergone coronary angiography (6-8). Furthermore, cohort studies demonstrated that blood concentrations of FABP4 in 495 non-T2DM subjects and 1,847 subjects without previous cardiovascular disease (CVD) in China were positively associated

*To whom correspondence should be addressed.

E-mail: gouda@u-shizuoka-ken.ac.jp with subsequent metabolic syndrome (MetS) and carotid atherosclerosis $(9,10)$. In addition, serum FABP4 concentrations were significant predictors for subsequent development of MetS in a 4-y follow-up for 465 subjects in South Korea (11). In Japan, serum concentrations of FABP4 were reported to be positively correlated with the body mass index (BMI), blood pressure (BP), low-density lipoprotein-cholesterol (LDL-C) level, homeostasis model assessment as an index of insulin resistance (HOMAIR) and mean left ventricular (LV) wall thickness, and negatively correlated with high-density lipoproteincholesterol (HDL-C) level, in a general population of 82 males and 108 females (12). Positive associations between blood concentrations of FABP4 and BP were strong in these studies. However, several studies have demonstrated that obesity is a strong risk factor for $\mathrm{BP}$ elevation (13). Whether a positive association between FABP4 concentrations and BP is independent of abdominal fat areas (AFAs) has not been investigated.

Blood concentrations of FABP4 were reported to be closely associated with LV diastolic dysfunction (which 
is related to $\mathrm{BP}$ dysfunction) in severely obese German subjects (14). In Japan, blood concentrations of FABP4 were found to be positively associated with $\mathrm{LV}$ diastolic dysfunction in a general population of 190 subjects (male/female: 82/108), and this association disappeared when BMI was inserted in multiple linear regression analyses (MLRA) (12). With respect to BP, it was reported that serum concentrations of FABP4 were higher in 30 Japanese subjects with essential hypertension than in 18 normotensive subjects, and that this association was independent of age, sex, and BMI (15). However, whether blood concentrations of FABP4 are positively associated with BP in a continuous fashion from a healthy state to a hypertensive state in a larger general population is not known. AFAs (total area of abdominal fat, visceral fat, or subcutaneous fat) can also be assessed by computed tomography (CT) to examine independent associations between blood concentrations of FABP4 and BP from abdominal fat.

A recent study demonstrated that serum concentrations of FABP were independently and positively associated with sarcopenic obesity as assessed by CT (16). However, a potential association between blood concentrations of FABP4 and abdominal fat in a general population by CT has not been assessed. Furthermore, whether a positive association between blood concentrations of FABP4 and BP is independent from AFA as assessed by CT has not been studied.

We examined independent associations of blood FABP4 concentrations with AFAs (total abdominal fat, visceral fat, and subcutaneous fat), which was assessed by CT, or BP by MLRA in 305 middle-aged Japanese males who were not taking medications for metabolic diseases.

\section{MATERIALS AND METHODS}

Ethical approval of the study protocol. The study protocol was approved by the Ethics Committee of the University of Shizuoka (Shizuoka, Japan) based on the Declaration of Helsinki. All subjects provided written informed consent for use of their personal information.

Subjects. We conducted a cross-sectional study of 305 middle-aged Japanese males (30-64 (mean \pm stan-

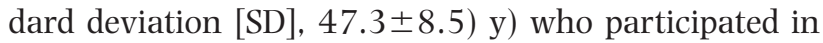
health check-ups at Shizuoka Health Promotion Center (Shizuoka, Japan) between July 2005 and March 2007.

We excluded subjects under treatment for: stroke; hypertension; cardiac disease; T2DM; hyperlipidemia; diseases of the liver, kidney, bladder or prostate gland; gout; psychiatric illness; cancer; ulcerative connectivetissue disease; adenoiditis; pancreatitis; rheumatism; accessory thyroid diseases; thyroid diseases; muscular atrophy; severe muscular asthenia; lung diseases (obstruction, emphysema, pneumonia, sarcoidosis, acute bronchitis, or interstitial lung disease); Behçet's disease; Graves' disease; Parkinsonian tremor; nephrosis syndrome; epilepsy; thrombocytopenia purpura; cholelithiasis; cholecystitis; cingulum; multiple myositis; distension of the common bile duct; systemic lupus erythematosus; hyperuricemia; pleurisy; and Hashimoto's

\section{thyroiditis}

Anthropometric data and blood samples were collected from each participant by trained medical staff. Smoking status was recorded as the number of cigarettes per day and duration of smoking (years). Self-reported physical activity was recorded as the number of exercise sessions undertaken each week (0-7). Dietary habits, energy intake, and alcohol intake during the previous month were assessed using a brief self-administered diet history questionnaire (BDHQ) $(17,18)$. The BDHQ was a fourpage, structured document with three sections: general dietary behavior and major cooking methods; frequency of consumption of food/drink; intake of five alcoholic beverages. Food and beverage items and their standard portion sizes were derived from a previously developed self-administered diet history questionnaire (DHQ) (1921), a 16-page structured questionnaire comprising seven sections. Estimated dietary intake of 48 food and beverage items, energy, and 42 nutrients was calculated using an ad hoc computer algorithm developed for the BDHQ. This algorithm was based primarily on the Standard Tables of Food Composition in Japan set by the Science and Technology Agency in 2000.

Measurements. After an overnight fast, BP [diastolic blood pressure (DBP) and systolic blood pressure (SBP)], height and weight were measured; blood samples were collected from each subject. BMI was calculated as weight in kilograms divided by the square of height in meters. Serum samples were stored at $-80^{\circ} \mathrm{C}$ for subsequent assays.

Levels of total cholesterol (TC), HDL-C, LDL-C, triacylglycerol (TG), fasting plasma glucose, hemoglobin A1c (HbA1c), aspartate aminotransferase (AST), alanine aminotransferase (ALT), $\gamma$-glutamyl-transpeptidase (GTP), creatinine, and high-sensitivity C-reactive protein (CRP; using the latex agglutination method) were measured, and the leukocyte count recorded. AFAs (total abdominal fat, visceral fat, and subcutaneous fat) were measured using CT.

Serum levels of FABP4 were measured using an enzyme-linked immunosorbent assay kit (Bio Vendor R\&D, Mordrice, Czech Republic) with calibration and quality control undertaken according to manufacturer instructions.

Statistical analyses. Clinical and biochemical data are the mean \pm standard deviation (SD), median, minimum, and maximum. Pearson product-moment correlation coefficient (PPMCC) analyses were used to calculate the correlations for FABP4 and each parameter as continuous variables. Correlation between FABP4 and categorized data to tertiles was calculated by PPMCC analyses. Previous studies have demonstrated that MLRA is useful for the selection of independent variables in various parameters $(22,23)$. Step-wise MLRA was done to examine the independent association between the blood concentration of FABP 4 and other parameters. Independent variables for the MLRA were selected using parameters with significant associations according to PPMCC analyses such as BMI, AFA (total abdominal, visceral, and subcutaneous fats), SBP, DBP, TC, HDL-C, TG, $\gamma$-GTP, 
Table 1. Physical characteristics, anthropometric characteristics, alcohol intake, and physical activity in Japanese males aged 30-64 y.

\begin{tabular}{|c|c|c|c|c|}
\hline Characterisitic $(n=305)$ & Mean & Median & Minimum & Maximum \\
\hline Age $(y)$ & $47.3 \pm 8.5$ & 47 & 30 & 64 \\
\hline Body height $(\mathrm{cm})$ & $170.1 \pm 5.3$ & 170.1 & 151.9 & 185.5 \\
\hline Body weight (kg) & $70.0 \pm 9.3$ & 69.2 & 47.9 & 108.3 \\
\hline $\operatorname{BMI}\left(\mathrm{kg} / \mathrm{m}^{2}\right)$ & $24.2 \pm 2.8$ & 23.9 & 17.4 & 34.4 \\
\hline Total abdominal fat area $\left(\mathrm{cm}^{2}\right)$ & $243.0 \pm 82.9$ & 235.2 & 82.2 & 520 \\
\hline Visceral fat area $\left(\mathrm{cm}^{2}\right)$ & $99.6 \pm 42.6$ & 94 & 20.3 & 235.7 \\
\hline Subcutaneous fat area $\left(\mathrm{cm}^{2}\right)$ & $143.4 \pm 55.4$ & 133.9 & 33.6 & 380.7 \\
\hline $\mathrm{SBP}(\mathrm{mmHg})$ & $117.0 \pm 14.0$ & 116 & 86 & 161 \\
\hline $\mathrm{DBP}(\mathrm{mmHg})$ & $74.9 \pm 11.1$ & 75 & 39 & 103 \\
\hline Heart rate (beats per minute) & $62.3 \pm 9.8$ & 61 & 38 & 98 \\
\hline $\mathrm{TC}(\mathrm{mg} / \mathrm{dL})$ & $214.3 \pm 31.8$ & 211 & 147 & 323 \\
\hline HDL-C (mg/dL) & $56.0 \pm 15.1$ & 55 & 30 & 113 \\
\hline LDL-C (mg/dL) & $133.3 \pm 28.1$ & 130 & 73 & 219 \\
\hline $\mathrm{TG}(\mathrm{mg} / \mathrm{dL})$ & $146.1 \pm 97.3$ & 124 & 30 & 1,101 \\
\hline Fasting plasma glucose (mg/dL) & $97.4 \pm 16.3$ & 95 & 77 & 287 \\
\hline HbAlc $(\%)$ & $5.57 \pm 0.68$ & 5.5 & 4.6 & 13.6 \\
\hline$\gamma$-GTP (U/L) & $52.5 \pm 45.5$ & 37 & 7 & 313 \\
\hline $\operatorname{AST}(\mathrm{U} / \mathrm{L})$ & $24.6 \pm 9.1$ & 22 & 13 & 80 \\
\hline $\operatorname{ALT}(\mathrm{U} / \mathrm{L})$ & $31.0 \pm 22.2$ & 25 & 9 & 218 \\
\hline Creatinine $(\mathrm{mg} / \mathrm{dL})$ & $0.86 \pm 0.11$ & 0.9 & 0.5 & 1.3 \\
\hline White blood cell count $\left(/ \mu \mathrm{L}, \times 10^{3}\right)$ & $6.24 \pm 1.53$ & 6.2 & 2.74 & 16.33 \\
\hline $\mathrm{CRP}(\mathrm{mg} / \mathrm{dL})$ & $0.18 \pm 0.28$ & 0.11 & 0 & 2.81 \\
\hline FABP4 (ng/mL) & $13.1 \pm 4.0$ & 12.61 & 4.35 & 25.77 \\
\hline \multicolumn{5}{|l|}{ Smoking } \\
\hline Number of cigarettes (number/d) & $11.3 \pm 12.8$ & 10 & 0 & 60 \\
\hline Duration of smoking $(\mathrm{y})$ & $10.4 \pm 12.5$ & 0 & 0 & 40 \\
\hline Physical activity (times/wk) & $2.0 \pm 2.5$ & 1 & 0 & 7 \\
\hline Alcohol intake $(\mathrm{g} / \mathrm{d})$ & $10.4 \pm 12.5$ & 21.0 & 0 & 187.9 \\
\hline Energy intake (kcal/d) & $2,202 \pm 541$ & 2,149 & 935 & 3,465 \\
\hline
\end{tabular}

ALT, alanine transferase; AST, aspartate aminotransferase; BMI, body mass index; CRP, C-reactive protein; DBP, diastolic blood pressure; $\gamma$-GTP, $\gamma$-glutamyl transpeptidase; FABP4, fatty acid binding protein 4; HbAlc, hemoglobin A1c; HDL-C, high-density lipoprotein-cholesterol; LDL-C, low-density lipoprotein-cholesterol; TC, total cholesterol; TG, triglyceride; SBP, systolic blood pressure; SD, standard deviation.

AST, ALT, creatinine, and white blood cell count. CRP was included in the MLRA because association between FABP4 and categorized CRP was significant. To avoid multicollinearity in the MLRA, total abdominal fat area and LDL-C were excluded in the MLRA because these are strongly associated with subcutaneous fat and total cholesterol, respectively ( $R$ for PPMCC analyses was $>0.8$ ) and associations of total abdominal fat area and LDL-C with FABP4 concentrations were weaker than those of subcutaneous fat and total cholesterol, respectively. Inclusion criteria and exclusion criteria for step-wise MLRA were under $p<0.02$ and $p \geq 0.02$, respectively. For all analyses, $p<0.05$ was considered significant. All statistical analyses were carried out using JMP v9.0 (SAS Institute Japan Co., Ltd., Tokyo, Japan).

\section{RESULTS}

Study subjects were all middle-aged Japanese males who were not taking medications for metabolic diseases. Characteristics of the subjects are shown in Table 1. Five, 39, 21, 24 and two subjects had fasting glucose
$>125 \mathrm{mg} / \mathrm{dL}, \mathrm{HbAlc}>6.0 \%$, SBP $>139 \mathrm{mmHg}, \mathrm{DBP}$ $>89 \mathrm{mmHg}$, and TG $>149 \mathrm{mg} / \mathrm{dL}$, respectively.

PPMCC analyses for serum concentrations of FABP4 as continuous variable of each parameter showed that FABP4 concentrations were positively associated with: BMI; AFAs (total abdominal, visceral, and subcutaneous); SBP; DBP; TC; LDL-C; TG; activities of $\gamma$-GTP, AST, and ALT; creatinine; white blood cell count; and levels of creatinine. $p$ for the correlation between blood concentrations of FABP4 and categorized data to tertiles for each parameter was determined. Significant positive associations were observed for BMI, AFAs (total abdominal, visceral, and subcutaneous), SBP, DBP, $\gamma$-GTP activity, AST activity, ALT activity, and white blood cell count as well as levels of creatinine, TC, LDL-C, TG and CRP. A significant negative association with HDL-C level was also noted (Table 2).

Next, we carried out step-wise MLRA for FABP4 using parameters as independent variables with significant association by PPMCC analyses. Areas of fats (visceral and subcutaneous), SBP, DBP, HDL-C, TG, creatinine 
Table 2. Relationship between parameters and serum concentrations of FABP4.

\begin{tabular}{|c|c|c|c|c|c|c|}
\hline & \multirow{2}{*}{$\mathrm{PPMCC}^{1}$} & & \multirow{2}{*}{$n$} & \multicolumn{2}{|c|}{ FABP4 (ng/dL) } & \multirow{2}{*}{$\begin{array}{c}p \text { for } \\
\text { categorized } \\
\text { data }^{2}\end{array}$} \\
\hline & & & & Mean & SD & \\
\hline \multirow[t]{3}{*}{ Age (y) } & & Low $(30-42)$ & 97 & 13.27 & 4.40 & \\
\hline & $r=-0.005$ & Middle (43-51) & 104 & 12.92 & 3.79 & $r=-0.023$ \\
\hline & $p=0.929$ & High (52-64) & 104 & 13.04 & 3.88 & $p=0.696$ \\
\hline \multirow[t]{3}{*}{$\mathrm{BMI}\left(\mathrm{kg} / \mathrm{m}^{2}\right)$} & & Low $(<23)$ & 105 & 10.78 & 2.96 & \\
\hline & $r=0.579$ & Middle $(\geq 23,<25)$ & 87 & 12.93 & 3.51 & $r=0.477$ \\
\hline & $p<0.001$ & High $(\geq 25)$ & 113 & 15.30 & 4.02 & $p<0.001$ \\
\hline \multirow[t]{3}{*}{ Total abdominal fat area $\left(\mathrm{cm}^{2}\right)$} & & Low $(<200)$ & 103 & 10.63 & 3.08 & \\
\hline & $r=0.644$ & Middle $(\geq 200,<280)$ & 109 & 12.86 & 3.13 & $r=0.537$ \\
\hline & $p<0.001$ & $\operatorname{High}(\geq 280)$ & 93 & 16.02 & 3.96 & $p<0.001$ \\
\hline \multirow[t]{3}{*}{ Area of visceral fat $\left(\mathrm{cm}^{2}\right)$} & & Low $(<75)$ & 97 & 11.04 & 3.66 & \\
\hline & $r=0.434$ & Middle $(\geq 75,<110)$ & 102 & 13.49 & 3.54 & $r=0.352$ \\
\hline & $p<0.001$ & $\operatorname{High}(\geq 110)$ & 106 & 14.52 & 4.03 & $p<0.001$ \\
\hline \multirow[t]{3}{*}{ Area of subcutaneous fat $\left(\mathrm{cm}^{2}\right)$} & & Low $(<120)$ & 114 & 10.74 & 2.84 & \\
\hline & $r=0.631$ & Middle $(\geq 120,<160)$ & 95 & 12.66 & 3.19 & $r=0.563$ \\
\hline & $p<0.0001$ & High $(\geq 160)$ & 96 & 16.24 & 3.88 & $p<0.001$ \\
\hline \multirow[t]{3}{*}{$\mathrm{SBP}(\mathrm{mmHg})$} & & Low $(<110)$ & 91 & 11.93 & 4.00 & \\
\hline & $r=0.175$ & Middle $(\geq 110,<120)$ & 97 & 13.57 & 3.76 & $r=0.158$ \\
\hline & $p=0.002$ & $\operatorname{High}(\geq 120)$ & 117 & 13.54 & 4.08 & $p=0.006$ \\
\hline \multirow[t]{3}{*}{ DBP (mmHg) } & & Low $(<70)$ & 101 & 11.92 & 3.81 & \\
\hline & $r=0.254$ & Middle $(\geq 70,<80)$ & 100 & 12.83 & 3.90 & $r=0.255$ \\
\hline & $p<0.001$ & $\operatorname{High}(\geq 80)$ & 104 & 14.41 & 3.96 & $p<0.001$ \\
\hline \multirow[t]{3}{*}{ Heart rate (beats per minute) } & & Low $(<59)$ & 109 & 12.79 & 4.23 & \\
\hline & $r=0.080$ & Middle $(\geq 59,<65)$ & 101 & 12.75 & 3.78 & $r=0.094$ \\
\hline & $p=0.162$ & High $(\geq 65)$ & 95 & 13.73 & 3.95 & $p=0.102$ \\
\hline \multirow[t]{3}{*}{$\mathrm{TC}(\mathrm{mg} / \mathrm{dL})$} & & Low $(<196)$ & 91 & 12.56 & 4.28 & \\
\hline & $r=0.143$ & Middle $(\geq 196,<220)$ & 86 & 12.28 & 3.90 & $r=0.158$ \\
\hline & $p=0.013$ & $\operatorname{High}(\geq 220)$ & 128 & 13.97 & 3.74 & $p=0.006$ \\
\hline \multirow[t]{3}{*}{ HDL-C (mg/dL) } & & Low $(<48)$ & 95 & 14.10 & 4.05 & \\
\hline & $r=-0.163$ & Middle $(\geq 48,<60)$ & 101 & 12.91 & 4.11 & $r=-0.179$ \\
\hline & $p=0.004$ & $\operatorname{High}(\geq 60)$ & 109 & 12.33 & 3.73 & $p=0.002$ \\
\hline \multirow[t]{3}{*}{ LDL-C (mg/dL) } & & Low $(<120)$ & 94 & 12.50 & 4.32 & \\
\hline & $r=0.136$ & Middle $(\geq 120,<140)$ & 99 & 12.69 & 3.74 & $r=0.145$ \\
\hline & $p=0.017$ & $\operatorname{High}(\geq 140)$ & 112 & 13.89 & 3.87 & $p=0.011$ \\
\hline \multirow[t]{3}{*}{$\mathrm{TG}(\mathrm{mg} / \mathrm{dL})$} & & Low $(<96)$ & 101 & 12.03 & 4.12 & \\
\hline & $r=0.193$ & Middle $(\geq 96,<150)$ & 95 & 12.75 & 3.71 & $r=0.238$ \\
\hline & $p<0.001$ & $\operatorname{High}(\geq 150)$ & 109 & 14.31 & 3.86 & $p=0.001$ \\
\hline \multirow[t]{3}{*}{ Fasting plasma glucose (mg/dL) } & & Low $(<92)$ & 101 & 12.87 & 4.10 & \\
\hline & $r=0.064$ & Middle $(\geq 92,<99)$ & 110 & 12.49 & 3.78 & $r=0.107$ \\
\hline & $p=0.262$ & High $(\geq 100)$ & 94 & 13.96 & 4.07 & $p=0.062$ \\
\hline \multirow[t]{3}{*}{ HbAlc $(\%)$} & & Low $(<5.3)$ & 80 & 13.31 & 3.73 & \\
\hline & $r=0.022$ & Middle $(\geq 5.3,<5.6)$ & 91 & 12.39 & 4.12 & $r=0.024$ \\
\hline & $p=0.709$ & $\operatorname{High}(\geq 5.6)$ & 134 & 13.39 & 4.08 & $p=0.676$ \\
\hline \multirow[t]{3}{*}{$\gamma$-GTP $(\mathrm{U} / \mathrm{L})$} & & Low $(<29)$ & 101 & 11.61 & 4.03 & \\
\hline & $r=0.127$ & Middle $(\geq 29,<51)$ & 98 & 13.38 & 3.56 & $r=0.263$ \\
\hline & $p=0.026$ & $\operatorname{High}(\geq 51)$ & 106 & 14.17 & 4.02 & $p<0.001$ \\
\hline
\end{tabular}


Table 2 (continued)

\begin{tabular}{|c|c|c|c|c|c|c|}
\hline & \multirow{2}{*}{$\mathrm{PPMCC}^{1}$} & & \multirow{2}{*}{$n$} & \multicolumn{2}{|c|}{ FABP4 (ng/dL) } & \multirow{2}{*}{$\begin{array}{c}p \text { for } \\
\text { categorized } \\
\text { data }^{2}\end{array}$} \\
\hline & & & & Mean & SD & \\
\hline \multirow[t]{3}{*}{ AST (U/L) } & & Low $(<20)$ & 89 & 11.53 & 3.27 & \\
\hline & $r=0.270$ & Middle $(\geq 20,<26)$ & 114 & 13.22 & 4.17 & $r=0.267$ \\
\hline & $p<0.001$ & $\operatorname{High}(\geq 26)$ & 102 & 14.25 & 4.03 & $p<0.001$ \\
\hline \multirow[t]{3}{*}{ ALT (U/L) } & & Low $(<21)$ & 104 & 11.08 & 3.15 & \\
\hline & $r=0.313$ & Middle $(\geq 21,<31)$ & 98 & 13.25 & 3.93 & $r=0.393$ \\
\hline & $p<0.001$ & $\operatorname{High}(\geq 31)$ & 103 & 14.90 & 3.99 & $p<0.001$ \\
\hline \multirow[t]{3}{*}{ Creatinine (mg/dL) } & & Low $(<0.9)$ & 147 & 12.56 & 3.86 & \\
\hline & $r=0.135$ & Middle $(\geq 0.9,<1)$ & 103 & 13.41 & 4.10 & $r=0.126$ \\
\hline & $p=0.019$ & $\operatorname{High}(\geq 1)$ & 55 & 13.81 & 4.14 & $p=0.028$ \\
\hline \multirow[t]{3}{*}{ White blood cell count $\left(/ \mu \mathrm{L}, \times 10^{3}\right)$} & & Low $(<5.6)$ & 108 & 12.17 & 3.95 & \\
\hline & $r=0.164$ & Middle $(\geq 5.6,<6.7)$ & 97 & 13.31 & 3.99 & $r=0.169$ \\
\hline & $p=0.004$ & $\operatorname{High}(\geq 6.7)$ & 100 & 13.81 & 3.95 & $p=0.003$ \\
\hline \multirow[t]{3}{*}{$\mathrm{CRP}(\mathrm{mg} / \mathrm{dL})$} & & Low $(<0.1)$ & 97 & 12.34 & 3.64 & \\
\hline & $r=-0.021$ & Middle $(\geq 0.1,<0.14)$ & 108 & 12.93 & 3.84 & $r=0.159$ \\
\hline & $p=0.713$ & High $(\geq 0.14)$ & 100 & 13.93 & 4.40 & $p=0.005$ \\
\hline \multirow[t]{3}{*}{ Number of cigarettes (number/d) } & & Low $(<1)$ & 143 & 13.03 & 4.15 & \\
\hline & $r=0.029$ & Middle $(\geq 1,<25)$ & 116 & 12.86 & 3.72 & $r=0.042$ \\
\hline & $p=0.618$ & $\operatorname{High}(\geq 25)$ & 46 & 13.73 & 4.28 & $p=0.465$ \\
\hline \multirow[t]{3}{*}{ Duration of smoking (y) } & & Low $(<1)$ & 155 & 13.05 & 4.14 & \\
\hline & $r=-0.0004$ & Middle $(\geq 1,<20)$ & 56 & 13.36 & 4.41 & $r=-0.010$ \\
\hline & $p=0.995$ & $\operatorname{High}(\geq 20)$ & 94 & 12.92 & 3.56 & $p=0.857$ \\
\hline \multirow[t]{3}{*}{ Physical activity (times per week) } & & Low $(<1)$ & 149 & 13.62 & 3.95 & \\
\hline & $r=-0.101$ & Middle $(\geq 1,<4)$ & 65 & 12.35 & 3.64 & $r=-0.112$ \\
\hline & $p=0.079$ & $\operatorname{High}(\geq 4)$ & 91 & 12.68 & 4.28 & $p=0.051$ \\
\hline \multirow[t]{3}{*}{ Alcohol intake (g/d) } & & Low $(<8)$ & 101 & 13.26 & 4.21 & \\
\hline & $r=-0.094$ & Middle $(\geq 8,<40)$ & 108 & 13.17 & 4.32 & $r=-0.051$ \\
\hline & $p=0.100$ & $\operatorname{High}(\geq 40)$ & 96 & 12.75 & 3.41 & $p=0.374$ \\
\hline \multirow[t]{3}{*}{ Energy intake (kcal/d) } & & Low $(<1,900)$ & 98 & 12.83 & 3.83 & \\
\hline & $r=0.008$ & Middle $(\geq 1,900,<2,400)$ & 98 & 13.51 & 4.16 & $r=0.005$ \\
\hline & $p=0.894$ & $\operatorname{High}(\geq 2,400)$ & 109 & 12.90 & 4.05 & $p=0.933$ \\
\hline
\end{tabular}

${ }^{1}$ PPMCCs were undertaken between FABP4 concentration and parameters as continuous variables.

${ }^{2} p$-values for association between between FABP4 and the tertiles of each parameter trend were calculated by PPMCC.

ALT, alanine transferase; AST, aspartate aminotransferase; BMI, body mass index; CRP, C-reactive protein; DBP, diastolic blood pressure; $\gamma$-GTP, $\gamma$-glutamyl transpeptidase; FABP4, fatty acid binding protein 4; HbAlc, hemoglobin A1c; HDL-C, high-density lipoprotein-cholesterol; LDL-C, low-density lipoprotein-cholesterol; TC, total cholesterol; TG, triglyceride; SBP, systolic blood pressure; SD, standard deviation; PPMCC, Pearson product-moment correlation coefficient.

and white blood cell count remained in the final model. A significant positive association was observed for areas of visceral and subcutaneous fats and DBP. Adjusted coefficient of determination $\left(R^{2}\right)$ was 0.450 and 0.456 in initial and final models, respectively (Table 3).

\section{DISCUSSION}

The present study focused on the associations among serum concentrations of FABP4, abdominal fat (measured by $\mathrm{CT}$ ) and BP. We found a positive association between blood concentrations of FABP4 and indicators of abdominal fat, such as BMI and the area of fat (total abdominal, visceral, and subcutaneous). Studies have demonstrated that blood concentrations of FABP4 are positively associated with the prevalence of obesity, insulin resistance, T2DM and/or carotid atherosclerosis in Asian countries $(6-8,24)$. Those studies, along with the present study, suggest that blood concentrations of FABP4 are positively associated with the prevalence of obesity in middle-aged Japanese males not taking medi- 
Table 3. Multiple regression analysis for tertiles of blood pressure using age, alcohol intake, energy intake, smoking, physical activity and serum FABP4 concentration as explanatory variables.

\begin{tabular}{|c|c|c|c|c|c|c|c|c|c|c|}
\hline \multirow[b]{2}{*}{$n=305$} & \multicolumn{5}{|c|}{$\begin{array}{c}\text { Initial model } \\
\text { (adjusted } R^{2}=0.450 \text { ) }\end{array}$} & \multicolumn{5}{|c|}{$\begin{array}{c}\text { Final model } \\
\text { (adjusted } R^{2}=0.456 \text { ) }\end{array}$} \\
\hline & $\beta$ & Standard $\beta$ & SE & $p$ & & $\beta$ & Standard $\beta$ & SE & $p$ & \\
\hline Intercept & -2.727 & 0.000 & 3.081 & 0.377 & & -2.028 & 0.000 & 2.307 & 0.380 & \\
\hline $\operatorname{BMI}\left(\mathrm{kg} / \mathrm{m}^{2}\right)$ & 0.012 & 0.008 & 0.122 & 0.921 & & & & & & \\
\hline Area of visceral fat $\left(\mathrm{cm}^{2}\right)$ & 0.015 & 0.163 & 0.005 & 0.003 & $* *$ & 0.016 & 0.169 & 0.005 & 0.001 & $* *$ \\
\hline Area of subcutaneous fat $\left(\mathrm{cm}^{2}\right)$ & 0.038 & 0.527 & 0.005 & $<0.0001$ & $* * *$ & 0.039 & 0.540 & 0.003 & $<0.0001$ & *** \\
\hline $\mathrm{SBP}(\mathrm{mmHg})$ & -0.026 & -0.091 & 0.020 & 0.188 & & -0.029 & -0.101 & 0.020 & 0.140 & \\
\hline DBP (mmHg) & 0.053 & 0.148 & 0.025 & 0.036 & $*$ & 0.056 & 0.155 & 0.025 & 0.027 & $*$ \\
\hline $\mathrm{TC}(\mathrm{mg} / \mathrm{dL})$ & 0.001 & 0.006 & 0.006 & 0.897 & & & & & & \\
\hline HDL-C (mg/dL) & 0.023 & 0.085 & 0.014 & 0.117 & & 0.020 & 0.075 & 0.013 & 0.123 & \\
\hline $\mathrm{TG}(\mathrm{mg} / \mathrm{dL})$ & 0.003 & 0.084 & 0.002 & 0.132 & & 0.003 & 0.079 & 0.002 & 0.099 & \\
\hline$\gamma$-GTP (U/L) & -0.005 & -0.051 & 0.004 & 0.306 & & & & & & \\
\hline AST (U/L) & 0.005 & 0.012 & 0.040 & 0.897 & & & & & & \\
\hline $\operatorname{ALT}(\mathrm{U} / \mathrm{L})$ & 0.006 & 0.035 & 0.017 & 0.709 & & & & & & \\
\hline Creatinine $(\mathrm{mg} / \mathrm{dL})$ & 4.700 & 0.131 & 1.569 & 0.003 & $* *$ & 4.753 & 0.133 & 1.537 & 0.002 & ** \\
\hline White blood cell count $\left(/ \mu \mathrm{L}, \times 10^{3}\right)$ & 0.258 & 0.099 & 0.122 & 0.035 & $*$ & 0.228 & 0.087 & 0.116 & 0.050 & * \\
\hline $\mathrm{CRP}(\mathrm{mg} / \mathrm{dL})$ & -0.669 & -0.047 & 0.637 & 0.294 & & & & & & \\
\hline
\end{tabular}

${ }^{*} p<0.05,{ }^{* *} p<0.01,{ }^{* * *} p<0.001$.

ALT, alanine transferase; AST, aspartate aminotransferase; BMI, body mass index; CRP, C-reactive protein; DBP, diastolic blood pressure; $\gamma$-GTP, $\gamma$-glutamyl transpeptidase; FABP4, fatty acid binding protein 4; HDL-C, high-density lipoproteincholesterol; TC, total cholesterol; TG, triglyceride; SBP, systolic blood pressure; SE, standard error.

cations for metabolic diseases.

We observed positive associations between serum concentrations of FABP4 and areas of fat (visceral and subcutaneous) (Table 3). It is known that insulin resistance and abdominal fat areas are related to inflammation status, and that enlarged adipocytes express insulin-resistant adipokines such as tumor necrosis factor (TNF)- $\alpha$ and resistin (25). It has been reported that blood concentrations of TNF- $\alpha$ are strongly positively associated with AFA (particularly visceral-fat area) (26). Additionally, it has been reported that the blood concentration of CRP (an inflammation marker measured frequently in health check-ups) is positively associated with an indicator for overweight/obesity such as areas of visceral adipose tissue (27). We observed a weak positive association between FABP4 and categorical data of CRP concentrations in blood (Table 2). Thus, insulin resistance and inflammation status could be related to FABP4 concentration. Whether the blood concentration of FABP4 is associated with insulin-resistant adipokines such as TNF- $\alpha$ and resistin in this population should be studied.

Interestingly, we found that serum concentrations of FABP4 were positively associated with SBP and DBP in middle-aged Japanese males not taking medications for metabolic diseases. Associations between serum concentrations of FABP4 and AFAs (visceral and subcutaneous) or DBP remained in MLRA using parameters as independent variables with a significant association by PPMCC analyses (Table 3). These results suggest that positive associations between blood concentrations of FABP4 and $\mathrm{BP}$ or parameters for abdominal fat are independent from other independent variables. FABP4 is expressed in adipocytes and macrophages (1). Thus, blood concentrations of FABP4 may indicate adiposity- and macrophage activation-associated development of hypertension and MetS-related diseases. Whether abdominal fats (subcutaneous fat and visceral fat), or macrophages contribute to blood concentrations of FABP4 should be investigated.

FABP4 was found to induce the development of insulin resistance and atherosclerosis in a model of atherosclerosis employing apolipoprotein-E deficient $\left(\mathrm{ApoE}^{-/-}\right)$ mice and mice with genetic disruption of FABP4 (28). In addition, injection of a chemical inhibitor of FABP4 into $\mathrm{ApoE}^{-1-}$ mice was found to inhibit the development of atherosclerosis (5). Thus, blood concentrations of FABP4 could be associated with subsequent hypertension-associated CVD. However, a causal association between blood concentrations of FABP4 and hypertension or subsequent development of CVD has not been demonstrated, and further studies are warranted.

Here, we observed that association between blood concentrations of FABP4 and DBP were stronger than with SBP (Tables 2 and 3). DBP has been reported to be a strong risk factor for the development of CVD in those aged $<50 \mathrm{y}$, whereas SBP is a strong risk factor in those aged $>60$ y (29). Our recent study demonstrated that blood concentrations of soluble E-selectin (an adhesion molecule that induces attachment of leukocytes to blood vessels and a risk factor of CVD development) in the same study cohort as the present study were strongly positively associated with DBP rather than SBP (30). Subjects in the present study had a mean 
age of $47.3 \pm 8.5 \mathrm{y}$. Thus, the stronger association of blood concentrations of FABP4 with DBP rather than with SBP in the present study could be because of the younger age of the participants and the earlier developmental stage of CVD. Whether there is an association between blood concentrations of FABP4 and DBP or SBP in elderly subjects warrants investigation. Furthermore, whether higher blood concentrations of FABP4 and/or soluble E-selectin in middle-aged Japanese males not taking medications for metabolic diseases leads to a higher prevalence of CVD should also be studied.

In MLRA, we found a positive and independent association between serum concentrations of FABP4 and creatinine levels among parameters tested in this population (Table 3). Blood concentrations of FABP4 were reported to be positively associated with renal dysfunction in T2DM patients without microalbuminuria (161 T2DM patients and 102 healthy non-T2DM controls) (31). Thus, higher blood concentrations of FABP4 in the general population and in subjects with T2DM may be associated with higher risks of development of nephropathy.

We showed an association between the blood concentration of FABP4 and areas of abdominal fats (visceral and subcutaneous), and between the blood concentration of FABP4 and DBP by MLRA. However, $R^{2}$ for the MLR analyses was relatively low (adjusted $R^{2}=0.456$ ) (Table 3). $R^{2}$ value of MLRA denotes the relevance of the multiple regression equation. Hence, it is difficult to predict the blood concentration of FABP4 by predictor variables using this equation, and other factors (including blood parameters and lifestyle factors) would also be related to the circulating concentration of FABP4. The reason why $R^{2}$ for the MLRA is relatively low could be that our study population was healthy subjects and subjects with low-grade metabolic disorders. Additionally, FABP4 expressed in the blood of the healthy individuals was relatively high. Thus, factors such as hormones and growth factors secreted steadily in the body would be related to maintenance of the blood concentration of FABP4. In addition, it is possible to make an association between the blood concentration of FABP4 and insulinresistant adipokines such as TNF- $\alpha$ and resistin. Thus, our results suggest that the association between the blood concentration of FABP4 and areas of abdominal fats (visceral and subcutaneous) and between the blood concentration of FABP4 and DBP are independent from other parameters in health check-ups determined in this study. However, other factors out of the parameters used in the present study could be related to the blood concentration of FABP4. Thus, other putative factors potentially related to the blood concentration of FABP4 should be identified. The association between the blood concentration of FABP4 and abdominal fat areas (total abdominal, visceral and subcutaneous), and between FABP4 and DBP by MLRAs with such putative factors should also also be examined.

The cross-sectional design of the present study could not be used to identify the causal relationship among blood concentrations of FABP4, AFAs, and BP. In par- ticular, whether FABP4 is a risk factor for the development of hypertension and/or CVD because blood levels of FABP4 are positively strongly associated with abdominal fat was not investigated. Additionally, the study cohort was relatively small. An independent association between blood levels of FABP4 and BP from AFAs was not tested in other regions in the general population or in subjects with hypertension or other metabolic diseases. Therefore, the association between blood concentrations of FABP4, hypertension, AFAs or subsequent elevation of BP and prevalence of CVD should be investigated in longitudinal studies in many regions of different countries.

In conclusion, we showed that blood concentrations of FABP4 are positively and independently associated with BP and AFAs (visceral and subcutaneous) in middle-aged Japanese males not taking medications for metabolic diseases.

\section{Acknowledgments}

We thank Drs. S. Sasaki and K. Murakami for providing the Brief Self-administered Diet History Questionnaire and for helpful discussions. This work was supported by the Global COE Program, the Ministry of Education, Culture, Sports, Science and Technology in Japan, and by a grant from the Takeda Science Foundation.

\section{Conflict of interest}

There are no conflicts of interest in this study.

\section{REFERENCES}

1) Gelsinger C, Tschoner A, Kaser S, Ebenbichler CF. 2010. Adipokine update-new molecules, new functions. Wien Med Wochenschr 160: 377-390.

2) Furuhashi M, Hotamisligil GS. 2008. Fatty acid-binding proteins: Role in metabolic diseases and potential as drug targets. Nat Rev Drug Discov 7: 489-503.

3) Boord JB, Maeda K, Makowski L, Babaev VR, Fazio S, Linton MF, Hotamisligil GS. 2004. Combined adipocyte-macrophage fatty acid-binding protein deficiency improves metabolism, atherosclerosis, and survival in apolipoprotein E-deficient mice. Circulation 110: 1492-1498.

4) Furuhashi M, Fucho R, Gorgun CZ, Tuncman G, Cao H, Hotamisligil GS. 2008. Adipocyte/macrophage fatty acid-binding proteins contribute to metabolic deterioration through actions in both macrophages and adipocytes in mice. J Clin Invest 118: 2640-2650.

5) Furuhashi M, Tuncman G, Gorgun CZ, Makowski L, Atsumi G, Vaillancourt E, Kono K, Babaev VR, Fazio S, Linton MF, Sulsky R, Robl JA, Parker RA, Hotamisligil GS. 2007. Treatment of diabetes and atherosclerosis by inhibiting fatty-acid-binding protein aP2. Nature 447: 959-965.

6) Rhee EJ, Lee WY, Park CY, Oh KW, Kim BJ, Sung KC, Kim BS. 2009. The association of serum adipocyte fatty acidbinding protein with coronary artery disease in Korean adults. Eur J Endocrinol 160: 165-172.

7) Bagheri R, Qasim AN, Mehta NN, Terembula K, Kapoor S, Braunstein S, Schutta M, Iqbal N, Lehrke M, Reilly MP. Relation of plasma fatty acid binding proteins 4 and 5 
with the metabolic syndrome, inflammation and coronary calcium in patients with type-2 diabetes mellitus. Am J Cardiol 106: 1118-1123.

8) Hong J, Gu W, Zhang Y, Yan Q, Dai M, Shi J, Zhai Y, Wang W, Li X, Ning G. 2011. Different association of circulating levels of adipocyte and epidermal fatty acidbinding proteins with metabolic syndrome and coronary atherosclerosis in Chinese adults. Atherosclerosis 217: 194-200.

9) Xu A, Tso AW, Cheung BM, Wang Y, Wat NM, Fong CH, Yeung DC, Janus ED, Sham PC, Lam KS. 2007. Circulating adipocyte-fatty acid binding protein levels predict the development of the metabolic syndrome: A 5-year prospective study. Circulation 115: 1537-1543.

10) Chow WS, Tso AW, $\mathrm{Xu} A$, Yuen MM, Fong CH, Lam TH, Lo SV, Tse HF, Woo YC, Yeung CY, Cheung BM, Lam KS. 2013. Elevated circulating adipocyte-fatty acid binding protein levels predict incident cardiovascular events in a community-based cohort: A 12-year prospective study. J Am Heart Assoc 2: e004176.

11) Park SE, Rhee EJ, Lee WY, Kim WJ, Yoo SH, Bae JC, Choi ES, Park CY, Oh KW, Park SW, Kim SW. 2012. The role of serum adipocyte fatty acid-binding protein on the development of metabolic syndrome is independent of pro-inflammatory cytokines. Nutr Metab Cardiovasc Dis 22: $525-532$.

12) Fuseya T, Furuhashi M, Yuda S, Muranaka A, Kawamukai M, Mita T, Ishimura S, Watanabe Y, Hoshina K, Tanaka M, Ohno K, Akasaka H, Ohnishi H, Yoshida H, Saitoh S, Shimamoto K, Miura T. 2014. Elevation of circulating fatty acid-binding protein 4 is independently associated with left ventricular diastolic dysfunction in a general population. Cardiovasc Diabetol 13: 126.

13) Arabshahi S, Busingye D, Subasinghe AK, Evans RG, Riddell MA, Thrift AG. 2014. Adiposity has a greater impact on hypertension in lean than not-lean populations: A systematic review and meta-analysis. Eur J Epidemiol 29: 311-324.

14) Baessler A, Lamounier-Zepter V, Fenk S, Strack C, Lahmann C, Loew T, Schmitz G, Bluher M, Bornstein SR, Fischer M. 2014. Adipocyte fatty acid-binding protein levels are associated with left ventricular diastolic dysfunction in morbidly obese subjects. Nutr Diabetes 4: e106.

15) Ota H, Furuhashi M, Ishimura S, Koyama M, Okazaki Y, Mita T, Fuseya T, Yamashita T, Tanaka M, Yoshida H, Shimamoto K, Miura T. 2012. Elevation of fatty acidbinding protein 4 is predisposed by family history of hypertension and contributes to blood pressure elevation. Am J Hypertens 25: 1124-1130.

16) Kim TN, Won JC, Kim YJ, Lee EJ, Kim MK, Park MS, Lee SK, Kim JM, Ko KS, Rhee BD. 2013. Serum adipocyte fatty acid-binding protein levels are independently associated with sarcopenic obesity. Diabetes Res Clin Pract 101: 210-217.

17) Murakami K, Mizoue T, Sasaki S, Ohta M, Sato M, Matsushita Y, Mishima N. 2008. Dietary intake of folate, other B vitamins, and $\omega-3$ polyunsaturated fatty acids in relation to depressive symptoms in Japanese adults. Nutrition 24: 140-147.

18) Kobayashi S, Murakami K, Sasaki S, Okubo H, Hirota N, Notsu A, Fukui M, Date C. 2011. Comparison of relative validity of food group intakes estimated by compre- hensive and brief-type self-administered diet history questionnaires against 16 d dietary records in Japanese adults. Public Health Nutr 14: 1200-1211.

19) Sasaki S, Yanagibori R, Amano K. 1998. Self-administered diet history questionnaire developed for health education: A relative validation of the test-version by comparison with 3-day diet record in women. J Epidemiol 8: 203-215.

20) Anonymous. 1998. Validity of a self-administered diet history questionnaire for assessment of sodium and potassium: Comparison with single 24-hour urinary excretion. Jpn Circ J 62: 431-435.

21) Sasaki S, Ushio F, Amano K, Morihara M, Todoriki O, Uehara Y, Toyooka E. 2000. Serum biomarker-based validation of a self-administered diet history questionnaire for Japanese subjects. J Nutr Sci Vitaminol 46: 285-296.

22) Maruyama C, Yoneyama M, Suyama N, Yoshimi K, Teramoto A, Sakaki Y, Suto Y, Takahashi K, Araki R, Ishizaka Y, Yamakado M, Teramoto T. 2008. Differences in serum phospholipid fatty acid compositions and estimated desaturase activities between Japanese men with and without metabolic syndrome. J Atheroscler Thromb 15: 306-313.

23) Mochizuki K, Misaki Y, Miyauchi R, Takabe S, Shimada M, Kuriki K, Ichikawa Y, Goda T. 2012. A higher rate of eating is associated with higher circulating interluekin$1 \beta$ concentrations in Japanese men not being treated for metabolic diseases. Nutrition 28: 978-983.

24) Koh JH, Shin YG, Nam SM, Lee MY, Chung CH, Shin JY. 2009. Serum adipocyte fatty acid-binding protein levels are associated with nonalcoholic fatty liver disease in type 2 diabetic patients. Diabetes Care 32: 147-152.

25) Bastard JP, Maachi M, Lagathu C, Kim MJ, Caron M, Vidal H, Capeau J, Feve B. 2006. Recent advances in the relationship between obesity, inflammation, and insulin resistance. Eur Cytokine Network 17: 4-12.

26) Scaglione R, Di Chiara T, Cariello T, Licata G. 2010. Visceral obesity and metabolic syndrome: Two faces of the same medal? Intern Emerg Med 5: 111-119.

27) Saijo Y, Kiyota N, Kawasaki Y, Miyazaki Y, Kashimura J, Fukuda M, Kishi R. 2004. Relationship between C-reactive protein and visceral adipose tissue in healthy Japanese subjects. Diabetes Obes Metab 6: 249-258.

28) Makowski L, Boord JB, Maeda K, Babaev VR, Uysal KT, Morgan MA, Parker RA, Suttles J, Fazio S, Hotamisligil GS, Linton MF. 2001. Lack of macrophage fatty-acidbinding protein aP2 protects mice deficient in apolipoprotein E against atherosclerosis. Nat Med 7: 699-705.

29) Franklin SS, Larson MG, Khan SA, Wong ND, Leip EP, Kannel WB, Levy D. 2001. Does the relation of blood pressure to coronary heart disease risk change with aging? The Framingham Heart Study. Circulation 103: 1245-1249.

30) Mochizuki K, Inoue S, Miyauchi R, Misaki Y, Shimada M, Kasezawa N, Tohyama K, Goda T. 2013. Plasma se-selectin level is positively correlated with neutrophil count and diastolic blood pressure in Japanese men. J Nutr Sci Vitaminol 59: 447-453.

31) Cabre A, Lazaro I, Girona J, Manzanares JM, Marimon F, Plana N, Heras M, Masana L. 2008. Plasma fatty acidbinding protein 4 increases with renal dysfunction in type 2 diabetic patients without microalbuminuria. Clin Chem 54: 181-187. 